\title{
GRAINE, $\gamma$-ray observations with a high angular resolving and polarization sensitive large-aperture emulsion telescope
}

\author{
Satoru Takahashi* \\ Kobe University \\ E-mail: satorudradix.h.kobe-u.ac.jp \\ for GRAINE collaboration \\ Aichi University of Education, \\ Institute of Space and Astronautical Science, Japan Aerospace Exploration Agency (ISAS/JAXA), \\ Kobe University, \\ Nagoya University, \\ Okayama University of Science
}

\begin{abstract}
We are developing GRAINE project, a $10-\mathrm{MeV}$ to $100-\mathrm{GeV}$ cosmic $\gamma$-ray observation project that uses an emulsion telescope with high angular resolution $\left(0.08^{\circ}\right.$ at 1 to $\left.2 \mathrm{GeV}\right)$ and polarization sensitivity, and a large aperture area $\left(\sim 10 \mathrm{~m}^{2}\right)$, by repeating long-duration balloon flights. Through various ground experiments, a balloon-borne experiment in 2011, and a balloon-borne experiment in 2015, the feasibility of cosmic $\gamma$-ray observations with a balloon-borne emulsion telescope was pioneered. Through scientific balloon-borne experiments, we can attempt to achieve the following: pioneering polarization observations for high-energy $\gamma$ rays from pulsars, active galactic nuclei, flares, and $\gamma$-ray bursts; direct probing of proton acceleration by $\pi^{0}$ feature detection and approaching an emission mechanism with a spatial structure for supernova remnants; resolving $\mathrm{GeV} \gamma$-ray excess at the galactic center region; probing new physics beyond the Planck scale by polarization observations with high-energy $\gamma$ rays propagating over cosmological distances; observing transient sources, e.g. $\gamma$-ray bursts and flares, with high photon statistics and high sensitivity; understanding emission mechanism of giant radio pulses from pulsars by searching for a $\gamma$-ray correlation; constraining intergalactic magnetic field by searching for $\mathrm{GeV}$ $\gamma$-ray pair halos. Developments in scientific balloon-borne experiments are ongoing.
\end{abstract}

35th International Cosmic Ray Conference

10-20 July, 2017

Bexco, Busan, Korea

\footnotetext{
* Speaker.
} 


\section{Introduction}

The observation of high-energy cosmic $\gamma$-rays provides direct information of high-energy phenomena in the universe. Fermi-LAT detected more than $3000 \gamma$-ray sources and is offering new insights [1 2]. On the other hand, past and current observations have some limitations. Improvements in an angular resolution and polarization sensitivity are one of keys for a breakthrough of the limitations.

We are developing GRAINE project, the project of a $10 \mathrm{MeV}-100 \mathrm{GeV}$ cosmic $\gamma$-ray observations with a precise $(0.08$ degree at $1-2 \mathrm{GeV})$ and polarization sensitive large aperture area $\left(\sim 10 \mathrm{~m}^{2}\right)$ emulsion telescope by repeating long duration balloon flights [3].

By various tests on the ground, a performance and feasibility of the emulsion $\gamma$-ray telescope were demonstrated using accelerator beams with $\gamma$-rays / electrons / muons and cosmic rays on the ground as well as atmospheric $\gamma$-rays at mountain height [4, 5, 6, 7, , , 9 .

In 2011, the first balloon-borne, emulsion $\gamma$-ray telescope experiment was successfully performed with a $125 \mathrm{~cm}^{2}$ aperture area and 4.3 hour flight duration on JAXA scientific ballooning at TARF Japan. By the flight data analysis, we demonstrated the feasibility of the balloon-borne emulsion $\gamma$-ray telescope experiment [10, 11].

In 2015, we had a JAXA scientific ballooning with a $3780 \mathrm{~cm}^{2}$ aperture area and 14.4 hour flight duration in Australia [12]13]. The telescope performance is being demonstrated by the flight data analysis 14, 15, 16, 17.

In 2018, we have a JAXA scientific ballooning with a $\sim 0.4 \mathrm{~m}^{2}$ aperture and $>\sim 18$ hour flight duration in Australia for a demonstration with a detection and imaging of a known $\gamma$-ray source, Vela pulsar. In 2021 or later, with a $10 \mathrm{~m}^{2}$ and $>24$ hour flight duration, we aim to start scientific observations [18].

\section{Prospects for scientific balloon-borne experiments}

A balloon experiment in Australia is an unique and hopeful site. Day-scale balloon experiments can be performed in Australia. Since Australia is located in the southern hemisphere, it is hoped that $\gamma$-ray sources in the southern sky can be observed (e.g., Vela, W44, and the galactic center). Due to the high geomagnetic rigidity at mid-latitudes, the charged particle background (as well as the neutral particle and $\gamma$-ray background) can be suppressed.

The 2015 balloon experiment was the first balloon experiment in Australia conducted by the current JAXA balloon group. The JAXA balloon group successfully performed the balloon experiment, and we performed a precursor experiment. The JAXA balloon group had a plan to continue the Australia balloon campaign. By enlarging the aperture area and flight duration, we attempt to start a scientific balloon experiment.

Due to its difficulty, the polarization of high-energy cosmic $\gamma$ rays has never been observed. This is a frontier in high-energy astrophysics. Pioneering research can be performed by an emulsion $\gamma$-ray telescope. We pioneer the polarization observation of pulsars, active galactic nuclei (AGNs), flares, and $\gamma$-ray bursts (GRBs), and approach the development of an emission mechanism and a structure of a magnetic field. Moreover, new physics searches can be performed beyond the Planck 
scale. Figure 1 shows the polarization sensitivity for a Vela pulsar. Significant observation exceeding $100 \%$ polarization can be started from around one transit (number of transits of a source in the field of view of the telescope with diurnal rotation (roughly corresponding to flight days)). By accumulating exposure, high polarization sensitivity can be achieved. Figure 2 shows the accumulated polarization sensitivity as a function of source flux. The Vela pulsar can be observed to degree of polarization of nearly $10 \%$. Polarization observation of other bright sources, pulsars, AGNs, flares, and GRBs, can also be achieved.
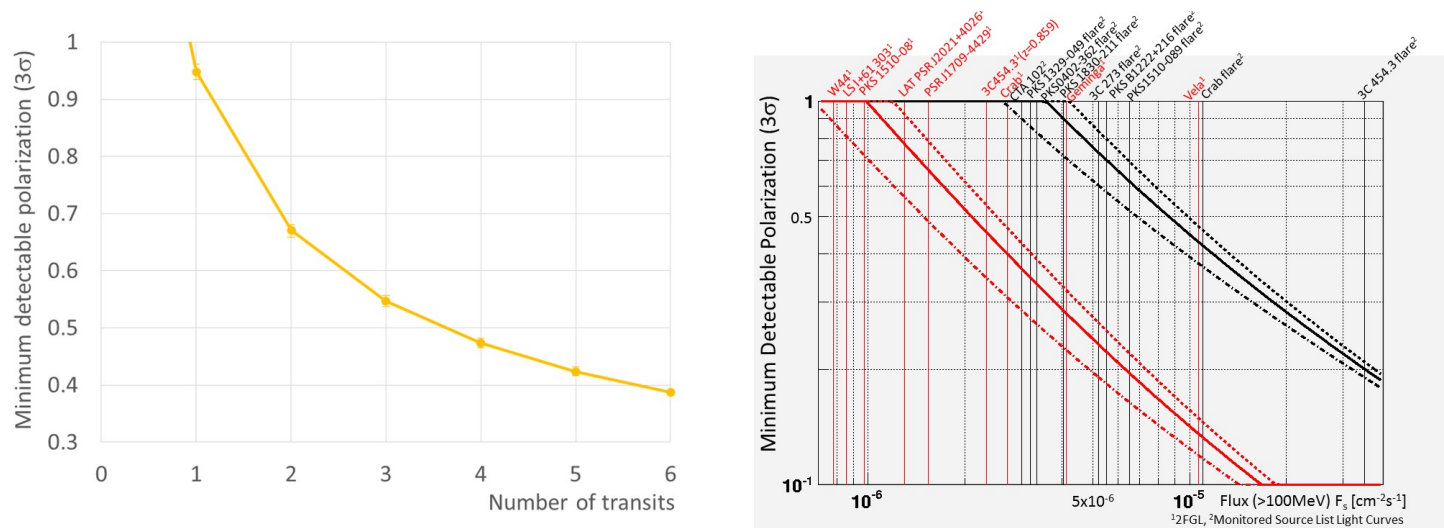

Figure 1: Polarization sensitivity for Vela pulsar ( $>$ Figure 2: Polarization sensitivity as a function of $200 \mathrm{MeV})$ as a function of the number of transits of source flux $(>100 \mathrm{MeV})$. Black curves indicate the a source in the field of view of the telescope with di- polarization sensitivity for $70 \mathrm{~m}^{2}$-days (aperture area urnal rotation (roughly corresponding to flight days) $\times$ flight days (roughly corresponding to a number of Aperture area: $10 \mathrm{~m}^{2}$, atmospheric depth: $5 \mathrm{~g} / \mathrm{cm}^{2}$, at transits)) accumulation. Red curves indicate the poAlice Springs. larization sensitivity for $70 \mathrm{~m}^{2}$-days (aperture area $\times$ flight days $) \times 10$ accumulation. The atmospheric depth is $5 \mathrm{~g} / \mathrm{cm}^{2}$. Dashed lines indicate a geomagnetic rigidity of $4.5 \mathrm{GV}$, and solid lines indicate a geomagnetic rigidity of $12 \mathrm{GV}$. Chain lines indicate a geomagnetic rigidity of $12 \mathrm{GV}$ with grain-by-grain precise measurement.

$\pi^{0}$ feature detection provides us with direct evidence of proton acceleration. Figure 3 shows the spectral energy distribution of supernova remnant (SNR) W44 observed by Fermi-LAT [19]. By detecting a decline below $200 \mathrm{MeV}$, the $\pi^{0}$ feature can be detected. The $\pi^{0}$ feature detection was reported as a Fermi-LAT collaboration. However, current measurements have a large systematic error below $200 \mathrm{MeV}$. The major factor was due to the uncertainty of the contamination of galactic diffuse $\gamma$ rays with an insufficient angular resolution below $200 \mathrm{MeV}$. Through observation using an emulsion $\gamma$-ray telescope with a high angular resolution, the contamination of galactic diffuse $\gamma$ rays can be drastically reduced $(<\sim 1 / 10)$. Thus, precise spectral measurements that can suppress systematic errors can be performed (Figure 3 (red points with error bars)). Precise validation of the $\pi^{0}$ feature for W44 can be performed. Systematic study of other SNRs is also possible. Moreover, precise low-energy measurements are expected to reveal new components, e.g., electron-induced components. In addition, by clarifying a spatial structure with a higher angular resolution, above 
$200 \mathrm{MeV}$, the emission mechanism can also be approached. Figure 4 shows the detection sensitivity for W44. Significant observation can be started from around two transits.
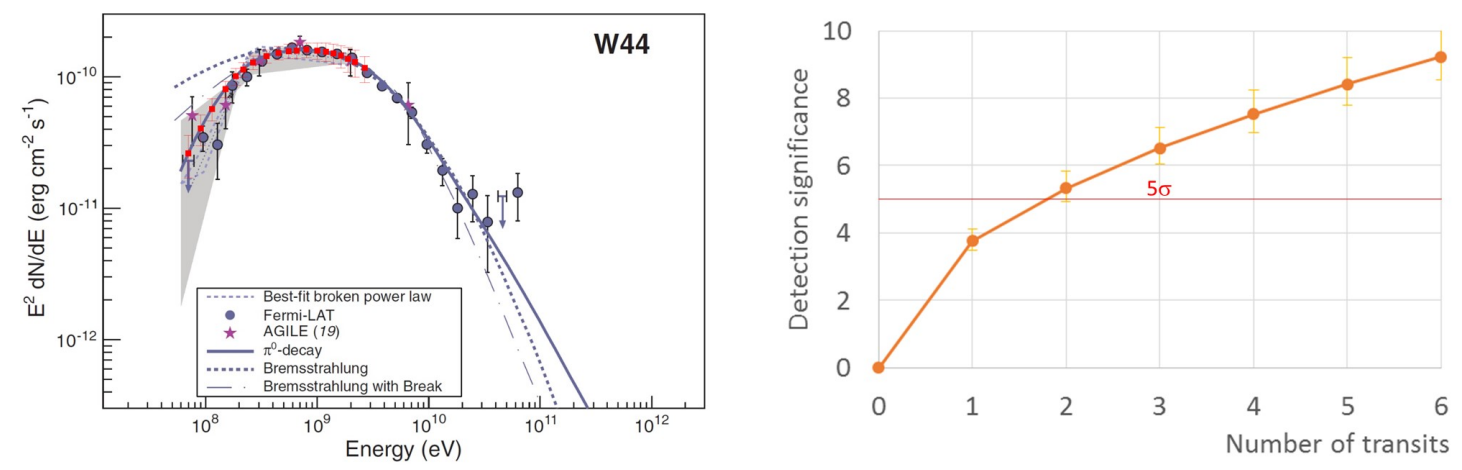

Figure 3: Spectral energy distribution of supernova Figure 4: Detection sensitivity for supernova remnant remnant W44 [19]. Red points with error bars indicate W44 (>200 MeV) by emulsion $\gamma$-ray telescope (apersimulated observations by the emulsion $\gamma$-ray tele- ture area: $10 \mathrm{~m}^{2}$, atmospheric depth: $5 \mathrm{~g} / \mathrm{cm}^{2}$, at Alice scope (accumulated: exposure $70 \mathrm{~m}^{2}$-transits (aper-Springs) as a function of the number of transits of a ture area $\times$ number of transits $) \times 10$, atmospheric source in the field of view of the telescope with diurdepth: $5 \mathrm{~g} / \mathrm{cm}^{2}$, at Alice Springs). nal rotation (roughly corresponding to flight days).

The $\mathrm{GeV} \gamma$-ray excess at the galactic center region has been reported by many groups using public data of Fermi-LAT. The GeV $\gamma$-ray excess was well explained as $\gamma$-ray-annihilating dark matter particles. On the other hand, the possibility of dense $\gamma$-ray sources at the galactic center region was considered. The $\mathrm{GeV} \gamma$-ray excess was also explained as $\gamma$-ray sources unresolved with the angular resolution of Fermi-LAT. Figure 5 shows simulations of $\mathrm{GeV} \gamma$-ray excess at the galactic center region with high angular resolution. The GeV $\gamma$-ray excess coming from the annihilation of dark matter particles or unresolved sources can be judged by the $\gamma$-ray intensity profile with a high angular resolution. Figure 6 shows the detection sensitivity for the galactic center region. Significant observation can be started from around one transit.

Using $\gamma$ rays propagating over cosmological distances, a new physics search can be performed beyond the Planck scale. Quantum gravity theories predict a violation of Lorentz invariance. The validation of fundamental symmetries beyond the Planck scale provides us with observational constraints for quantum gravity theories. By checking the energy dependence of the arrival timing of $\gamma$ rays from GRBs by Fermi-LAT, Lorentz invariance was proven around the Planck scale [22]. By polarization observation from GRBs by GAP (70 to $300 \mathrm{keV}$ ), CPT invariance was proven beyond the Planck scale [23]. The degree of $C P T$ violation (here, the rotation angle of the polarization vector) is proportional to the square of the photon energy. By polarization observation from distant AGNs and GRBs with high-energy $\gamma$ rays (e.g., $>100 \mathrm{MeV}$ ) by emulsion $\gamma$-ray telescope, very precise (five orders of magnitude better) validation can be performed.

Transient sources, e.g. GRBs and flares, can be observed by emulsion $\gamma$-ray telescope with a large collection area (high photon statistics), wide field of view (above $2.2 \mathrm{sr}(17.5 \%$ of all sky)), high angular resolution, polarization sensitivity and dead time free. Figure 7 shows sensitivities to transient sources by emulsion $\gamma$-ray telescope. Emulsion $\gamma$-ray telescope can observe an unexplored 
GRAINE, $\gamma$-ray observations with a high angular resolving and polarization sensitive large-aperture emulsion telescope Satoru Takahashi
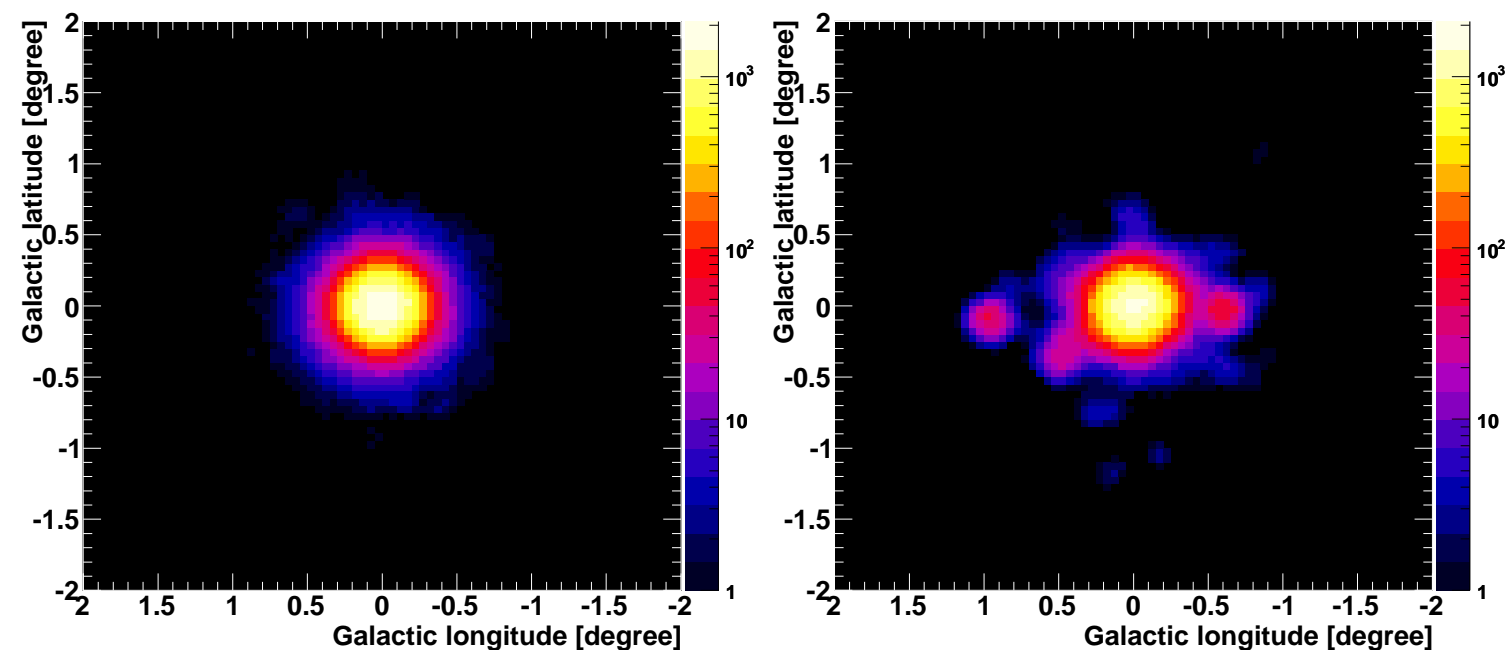

Figure 5: Simulations of the $\mathrm{GeV} \gamma$-ray excess $(>1 \mathrm{GeV})$ at the galactic center region (color scale: counts / $\left.(0.05 \text { degree })^{2}\right)$ with a high angular resolution $(0.1$ degree at $68 \%$ containment (corresponding to the angular resolution of the emulsion $\gamma$-ray telescope at $1 \mathrm{GeV}$ ), 4,200 $\mathrm{m}^{2}$-hours). (Left) Scenario of the annihilation of dark matter particles (spatial distribution of $\gamma$-ray intensity $\propto$ (distance from galactic center) ${ }^{-2.4}, \gamma$-ray energy spectrum [20]). (Right) Scenario of unresolved sources (luminosity function of the millisecond pulsar [21, spatial distribution of $\gamma$-ray sources $\propto(\text { distance from galactic center })^{-2.4}$ ).

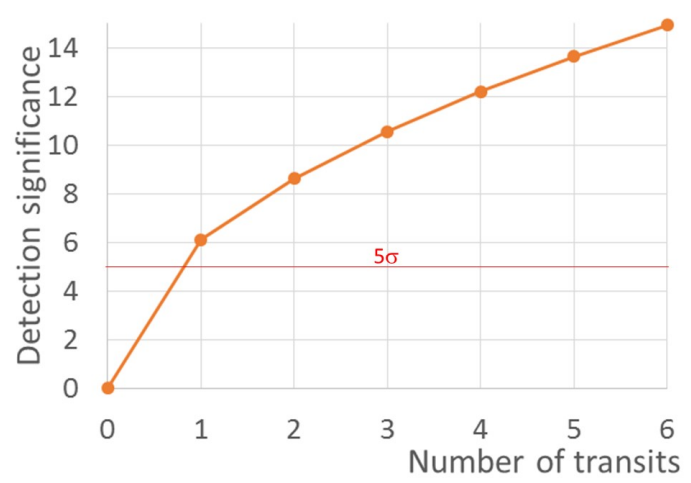

Figure 6: Detection sensitivity for the galactic center region $(>1 \mathrm{GeV})$ by emulsion $\gamma$-ray telescope (aperture area: $10 \mathrm{~m}^{2}$, atmospheric depth: $5 \mathrm{~g} / \mathrm{cm}^{2}$, at Alice Springs) as a function of the number of transits of a source in the field of view of the telescope with diurnal rotation (roughly corresponding to flight days).

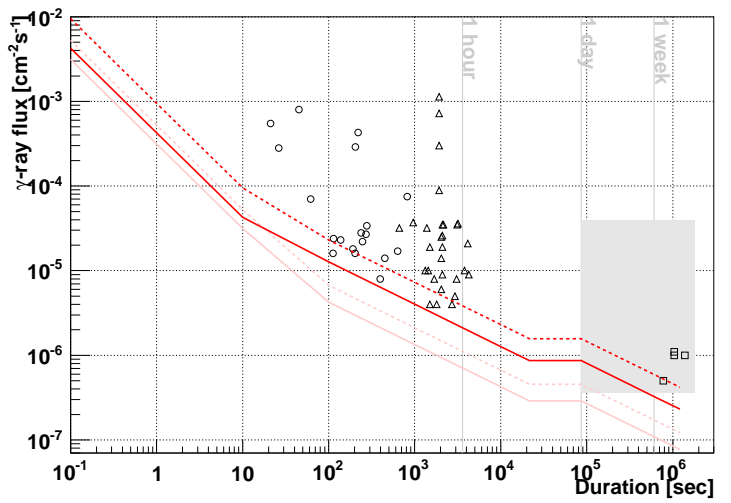

Figure 7: Flux sensitivities to transient sources by emulsion $\gamma$-ray telescope as a function of a duration $\left(10 \mathrm{~m}^{2}\right.$ aperture area, $5 \mathrm{~g} / \mathrm{cm}^{2}$ atmospheric depth, above $5 \sigma$ significance, above 5 photons). Red lines show sensitivities above $100 \mathrm{MeV}$. Pink lines show sensitivities above $200 \mathrm{MeV}$. Solid lines show a normal incidence. Dashed lines show a 45 degree zenith incidence. Each symbol and shade show transient sources detected by Fermi-LAT (circle: GRB (2008 Aug - 2011 Aug, $100 \mathrm{MeV}$ - $10 \mathrm{GeV}, T_{90, L A T}$ ) [24], triangle: solar flare (2008 Aug - $2012 \mathrm{Aug}, 100 \mathrm{MeV}$ - $10 \mathrm{GeV})$ [25], square: nova $(>100 \mathrm{MeV})$ [26], shaded area: flare (2FAV, 7.4 years, 4547 flares, 100 $\mathrm{MeV}-800 \mathrm{MeV}$ ) [27). 
region with a duration below several tens second. The sensitivity with a duration above several tens second can be performed to be comparable to Fermi-LAT's one. Observed rate of transient sources by Fermi-LAT was 638 transient sources per year (mainly flares). 1.7 transient sources per flight day can be observed by emulsion $\gamma$-ray telescope with a high statistics energy spectrum, time variation and polarization sensitivity.

Other topics are also being considered. A search for a $\gamma$-ray correlation with giant radio pulses from pulsars may provide an understanding of its emission mechanism [28]. It is easy for the balloon-borne emulsion $\gamma$-ray telescope to obtain simultaneous observation time with a radio telescope. And the emulsion $\gamma$-ray telescope can observe with a large collection area and dead time free. An observation of $\mathrm{GeV} \gamma$-ray pair halos provides constraints on intergalactic magnetic field [29]. The emulsion $\gamma$-ray telescope can observe with high angular resolution $(0.08$ degree at $1-2$ $\mathrm{GeV}$ ) for an individual source.

\section{Development of scientific balloon-borne experiments}

In order to achieve a $10 \mathrm{~m}^{2}$ aperture area, units of the emulsion $\gamma$-ray telescope are deployed. The allowable balloon-borne payload is $\sim 2$ tons. In a $10 \mathrm{~m}^{2}$ aperture area deployment, the weight of the emulsion films (100 stacked films) is 1 ton. The multi-stage shifter in GRAINE 2015 (open area: $2,500 \mathrm{~cm}^{2}$, weight: $65 \mathrm{~kg}$ ) has a weight of 2.6 tons in a $10 \mathrm{~m}^{2}$ aperture area deployment. This is not acceptable for a balloon-borne experiment. Thus, drastically lightening of the multi-stage shifter is essential in order to achieve a $10 \mathrm{~m}^{2}$ aperture area. In addition, in order to achieve a flight duration with more than one transit, a long-duration capability is needed for the multi-stage shifter. Long-duration capability requires narrow gaps between a higher number of stages.

In order to achieve a large aperture area and long duration capability, a conventional model of a multi-stage shifter (stage-plate-driven model) was reviewed on a zero basis, and a model with a new mechanism (roller-driven model) was invented (Figure 87. A roller-driven model has no stage plates or guide rail, so it is lighter. Moreover, as a result of the embedded structure (IRECO structure), narrow gaps between stages and an increasing number of stages can be realized. In addition, it is easy to enlarge the aperture area in a unit. Thus, the number of deployed units can be reduced while maintaining the total aperture area in the deployment. As a result, the total weight can be reduced.

Based on the invention, a prototype of the roller-driven model was constructed by Mitaka Kohki Co., Ltd. from August 2014 (Figure 9). Currently, working and performance tests are ongoing and a required accuracy of within $1 \mu \mathrm{m}$ is being obtained. In addition, a $10 \mathrm{~m}^{2}$ aperture area in the deployment based on the prototype has a weight of 0.4 tons, which is 6.5 times lighter that the conventional model (2.6 tons), resulting in a drastic weight reduction.

For an emulsion analysis with a large area [A $10 \mathrm{~m}^{2}$ aperture area corresponds to a $\sim 1000 \mathrm{~m}^{2}$ emulsion area $\left(10 \mathrm{~m}^{2} \times \sim 100\right.$ films).], a latest emulsion scanning system, Hyper Track Selector, achieved a $0.5 \mathrm{~m}^{2} /$ hour scanning speed [30]. Moreover, a faster scanning system is being developed with a $2.5 \mathrm{~m}^{2} /$ hour scanning speed. A data processing will follow the emulsion scanning.

\section{References}

[1] W. B. Atwood et al., ApJ. 697 (2009) 1071. 


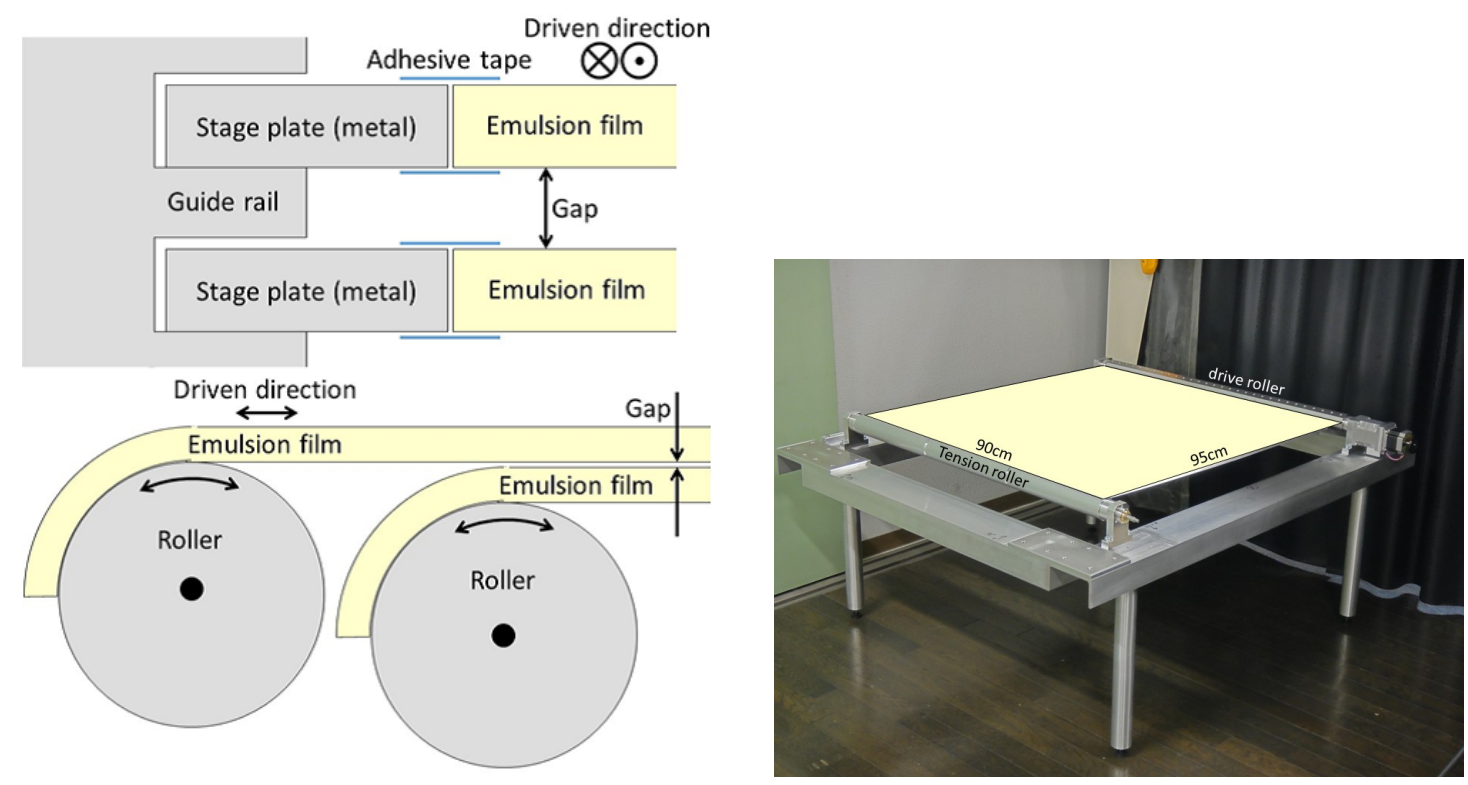

Figure 8: (Top) Conventional model (stage-plate- Figure 9: A prototype of the new model (roller-driven driven model). (Bottom) New model (roller-driven model).

model).

[2] F. Acero et al., ApJS 21823 (2015).

[3] S. Aoki et al., arXiv:1202.2529.

[4] S. Takahashi et al., Nucl. Instrum. Meth. A, 620 (2010) 192-195.

[5] S. Takahashi, Ph.D. Thesis, Nagoya University, Japan, 2011 (in Japanese).

[6] S. Takahashi et al., Proc. 33rd Int. Cosmic Ray Conference, 228 (2013).

[7] K. Ozaki, Ph.D. Thesis, Kobe University, Japan, 2016 (in Japanese).

[8] K. Ozaki et al., Nucl. Instrum. Meth. A 833 (2016) 165.

[9] H. Kawahara, Master Thesis, Nagoya University, Japan, 2015 (in Japanese).

[10] S. Takahashi et al., Prog. Theor. Exp. Phys. 043 H01 (2015).

[11] H. Rokujo et al., Nucl. Instrum. Meth. A 701, 127 (2013).

[12] S. Takahashi et al., Prog. Theor. Exp. Phys. 073F01 (2016).

[13] K. Ozaki et al., JINST 10 P12018 (2015).

[14] F. Mizutani et al., Proc. 31st Int. Symposium on Space Technology and Science (2017).

[15] A. Iyono et al., Proc. 35th Int. Cosmic Ray Conference (2017).

[16] H. Kawahara et al., Proc. 35th Int. Cosmic Ray Conference (2017).

[17] H. Rokujo et al., Proc. 35th Int. Cosmic Ray Conference (2017).

[18] S. Takahashi et al., Adv. Space Res. (submitted to Special Issue: Origins of Cosmic Rays).

[19] M. Ackermann et al., Science 339, 807 (2013). 
[20] T. Daylan et al., Physics of the Dark Universe 12 (2016) 1-23.

[21] D. Hooper et al., JCAP03 (2016) 049.

[22] A. A. Abdo et al., Nature 462, 331-334 (2009).

[23] K. Toma et al., Phys. Rev. Lett. 109, 241104 (2012).

[24] M. Ackermann et al., ApJS 20911 (2013).

[25] M. Ackermann et al., ApJ 78715 (2014).

[26] M. Ackermann et al., Science 3456196 (2014).

[27] S. Abdollahi et al., arXiv:1612.03165.

[28] A. Bilous et al., ApJ 728110 (2011).

[29] W. Chen et al., Phys. Rev. Lett. 115, 211103 (2015).

[30] M. Yoshimoto, T. Nakano, R. Komatani and H. Kawahara, submitted to Prog. Theor. Exp. Phys. (arXiv:1704.06814). 